\title{
MAXIMUM PRINCIPLES FOR A FAMILY OF NONLOCAL BOUNDARY VALUE PROBLEMS
}

\author{
PAUL W. ELOE
}

Received 21 October 2003 and in revised form 16 February 2004

We study a family of three-point nonlocal boundary value problems (BVPs) for an $n$ thorder linear forward difference equation. In particular, we obtain a maximum principle and determine sign properties of a corresponding Green function. Of interest, we show that the methods used for two-point disconjugacy or right-disfocality results apply to this family of three-point BVPs.

\section{Introduction}

The disconjugacy theory for forward difference equations was developed by Hartman [15] in a landmark paper which has generated so much activity in the study of difference equations. Sturm theory for a second-order finite difference equation goes back to Fort [12], which also serves as an excellent reference for the calculus of finite differences. Hartman considers the $n$ th-order linear finite difference equation

$$
P u(m)=\sum_{j=0}^{n} \alpha_{j}(m) u(m+j)=0,
$$

$\alpha_{n} \alpha_{0} \neq 0, m \in I=\{a, a+1, a+2, \ldots\}$. To illustrate the analogy of (1.1) to an $n$ th-order ordinary differential equation, introduce the finite difference operator $\Delta$ by

$$
\begin{gathered}
\Delta u(m)=u(m+1)-u(m), \quad \Delta^{0} u(m) \equiv u(m), \\
\Delta^{i+1} u(m)=\Delta\left(\Delta^{i} u\right)(m), \quad i \geq 1 .
\end{gathered}
$$

Clearly, $P$ can be algebraically expressed as an $n$ th-order finite difference operator.

Let $m_{1}, b$ denote two positive integers such that $n-2 \leq m_{1}<b$. In this paper, we assume that $a=0$ for simplicity, and we consider a family of three-point boundary conditions of the form

$$
u(0)=0, \ldots, u(n-2)=0, \quad u\left(m_{1}\right)=u(b)
$$


Clearly, the boundary conditions (1.3) are equivalent to the boundary conditions

$$
\Delta^{i} u(0)=0, \quad i=0, \ldots, n-2, \quad u\left(m_{1}\right)=u(b)
$$

There is a current flurry to study nonlocal boundary conditions of the type described by (1.3). In certain sectors of the literature, such boundary conditions are referred to as multipoint boundary conditions. Study was initiated by Il'in and Moiseev [16, 17]. These initial works were motivated by earlier work on nonlocal linear elliptic boundary value problems (BVPs) (see, e.g., [3, 4]). Gupta and coauthors have worked extensively on such problems; see, for example, $[13,14]$. Lomtatidze [18] has produced early significant work. We point out that Bobisud [5] has recently developed a nontrivial application of such problems to heat transfer. For the rest of the paper, we will use the term nonlocal boundary conditions, initiated by Il'in and Moiseev [16, 17].

We motivate this paper by first considering the equation

$$
P u(m)=\Delta^{n} u(m)=0, \quad m=0, \ldots, b-n .
$$

In this preliminary discussion, we employ the natural family of polynomials, $m^{(k)}=$ $m(m-1) \cdots(m-k+1)$ so that $\Delta m^{(k)}=k m^{(k-1)}$.

A Green function, $G\left(m_{1}, m, s\right)$ for the BVP (1.5), (1.3) exists for $\left(m_{1}, m, s\right) \in\{n-$ $2, \ldots, b-1\} \times\{0, \ldots, b\} \times\{0, \ldots, b-n\}$. It can be constructed directly and has the form

$$
G\left(m_{1} ; m, s\right)= \begin{cases}\frac{a\left(m_{1} ; s\right) m^{(n-1)}}{(n-1) !}, & 0 \leq m \leq s \leq b-n, \\ \frac{a\left(m_{1} ; s\right) m^{(n-1)}+(m-(s+1))^{(n-1)}}{(n-1) !}, & 0 \leq s+1 \leq m \leq b\end{cases}
$$

where

$$
\begin{aligned}
& a\left(m_{1} ; s\right)=-\frac{(b-(s+1))^{(n-1)}}{b^{(n-1)}-m_{1}^{(n-1)}}, \quad m_{1} \leq s, \\
& a\left(m_{1} ; s\right)=-\frac{(b-(s+1))^{(n-1)}-\left(m_{1}-(s+1)\right)^{(n-1)}}{b^{(n-1)}-m_{1}^{(n-1)}}, \quad s+1 \leq m_{1} .
\end{aligned}
$$

Associated with the BVP (1.5), (1.3) are two extreme cases. At $m_{1}=n-2$, we have the boundary conditions

$$
u(0)=0, \ldots, u(n-2)=0, \quad u(n-2)=u(b),
$$

which are equivalent to the two-point conjugate conditions [15]

$$
u(0)=0, \ldots, u(n-2)=0, \quad u(b)=0 .
$$

At $m_{1}=b-1$, we have the boundary conditions

$$
u(0)=0, \ldots, u(n-2)=0, \quad u(b-1)=u(b),
$$


which are equivalent to the two-point "in between conditions" [9]

$$
u(0)=0, \ldots, u(n-2)=0, \quad \Delta u(b-1)=0 .
$$

The following inequalities have been previously obtained [10, 15]:

$$
0>G(n-2 ; m, s)>G(b-1 ; m, s)
$$

$(m, s) \in\{n-1, \ldots, b\} \times\{0, \ldots, b-n\}$.

The following theorem is obtained directly from the representation (1.6) of $G\left(m_{1}\right.$; $m, s)$.

Theorem 1.1. $G\left(m_{1} ; m, s\right)$ is decreasing as a function of $m_{1}$; that is,

$$
0 \geq G\left(m_{1} ; m, s\right)>G\left(m_{1}+1 ; m, s\right)
$$

$\left(m_{1}, m, s\right) \in\{n-2, \ldots, b-2\} \times\{n-1, \ldots, b\} \times\{0, \ldots, b-n\}$. The first inequality is strict, except in the conjugate case, $m_{1}=n-2$, at $m=b$.

The purpose of this paper is to obtain Theorem 1.1 for a more general finite difference equation, $P u(m)=0$. Note that even for the specific BVP (1.5), (1.3), the calculations to show that $G$ is decreasing in $m_{1}$ are tedious. The method exhibited in the next section allows one to bypass the tedious calculations. We will need to assume a condition that implies disconjugacy. We will then argue that similar results are obtained if the nonlocal boundary condition has the form

$$
\Delta^{j} u\left(m_{1}\right)=\Delta^{j} u(b-j), \quad j \in\{0, \ldots, n-1\}
$$

The similar results will be valid if we assume that $P u(m)=0$ is right-disfocal [2].

\section{A general disconjugate equation}

Hartman [15] defined the disconjugacy of (1.1) on $I=\{0, \ldots, b\}$. First recall the definition of a generalized zero [15]. $m=0$ is a generalized zero of $u$ if $u(0)=0 . m>0$ is a generalized zero of $u$ if $u(m)=0$, or there exists an integer $k \geq 1$ such that $m-k \geq 0$, $u(m-k+1)=\cdots=u(m-1)=0$, and $(-1)^{k} u(m-k) u(m)>0$. Then (1.1) is disconjugate on $I$ if $u$ is a solution of (1.1) on $I$ and that $u$ has at least $n$ generalized zeros on $I$ implies that $u \equiv 0$ on $I$. A condition related to and stronger than disconjugacy is that of right-disfocality $[1,8] ;(1.1)$ is right-disfocal on $I$ if $u$ is a solution of (1.1) on $I$ and that $\Delta^{j} u$ has a generalized zero at $s_{j}, 0 \leq s_{0} \leq s_{1} \leq \cdots \leq s_{n-1} \leq b-n+1$, implies that $u \equiv 0$ on $I$. For this particular paper, a concept of right $(n-1 ; j)$ disfocality would be appropriate; $(1.1)$ is right $(n-1 ; j)$ disfocal on $I$ if $u$ is a solution of $(1.1)$ on $I$ and that $u$ has at least $n-1$ generalized zeros at $s_{0}, \ldots, s_{n-2}, \Delta^{j} u$ has a generalized zero at $s_{n-1}$, $\max \left\{s_{0}, \ldots, s_{n-2}\right\} \leq s_{n-1} \leq b-j$, imply that $u \equiv 0$ on $I$.

Hartman [15] showed the equivalence of disconjugacy and a Frobenius factorization in the discrete case; in particular, $P u=0$ is disconjugate on $\{0, \ldots, b\}$ if and only if there 
204 Nonlocal boundary value problems

exist positive functions $v_{i}$ defined on $\{0, \ldots, b-i+1\}$ such that

$$
P u(m)=\left(\frac{1}{v_{n+1}}\right) \Delta\left(\left(\frac{1}{v_{n}}\right) \Delta\left(\cdots \Delta\left(\left(\frac{1}{v_{2}}\right) \Delta\left(\frac{u}{v_{1}}\right)\right) \cdots\right)\right)(m)
$$

$m \in\{0, \ldots, b-n\}$. Define quasidifferences

$$
\begin{aligned}
P_{0} u(m) & =\left(\frac{u}{v_{1}}\right)(m), \\
P_{j} u(m) & =\left(\frac{1}{v_{j+1}}\right) \Delta\left(\left(\frac{1}{v_{j}}\right) \Delta\left(\cdots \Delta\left(\left(\frac{1}{v_{2}}\right) \Delta\left(\frac{u}{v_{1}}\right)\right) \cdots\right)\right)(m) \\
& =\left(\frac{1}{v_{j+1}}\right) \Delta\left(P_{j-1} u\right)(m),
\end{aligned}
$$

$m \in\{0, \ldots, b-j\}, j=0, \ldots, n$. We will now consider a family of nonlocal boundary conditions of the form

$$
P_{j} u(0)=0, \quad j=0, \ldots, n-2, \quad P_{0} u\left(m_{1}\right)=P_{0} u(b) .
$$

We will remind the reader of a version of Rolle's theorem.

Lemma 2.1. Let $u$ be a sequence of reals defined on a set of integers. If $P_{j} u$ has generalized zeros at $\mu_{1}$ and $\mu_{2}$, where $\mu_{1}<\mu_{2}$, then $P_{j+1} u$ has a generalized zero in $\left\{\mu_{1}, \ldots, \mu_{2}-1\right\}$.

Proof. Hartman [15] proved that $v_{j+2} P_{j+1} u$ has a generalized zero in the set $\left\{\mu_{1}, \ldots, \mu_{2}-\right.$ 1 \}. The lemma follows since $v_{j+2}$ is positive.

Theorem 2.2. Assume that $P u=0$ is right $(n-1 ; 1)$ disfocal on $\{0, \ldots, b\}$. Then there exists a uniquely determined Green function $G\left(m_{1} ; m, s\right)$ for the BVPs (1.1), (2.3).

Proof. Let $v$ denote the solution of the initial value problem (IVP) (1.1), satisfying initial conditions

$$
P_{j} v(0)=0, \quad j=0, \ldots, n-2, \quad P_{n-1} v(0)=1 .
$$

Let $\chi(m, s)$ denote the Cauchy function for $(1.1)$; that is, $\chi$, as a function of $m$, is the solution of the IVP (1.1), with the initial conditions

$$
\chi(s+1+j, s)=0, \quad j=0, \ldots, n-2, \quad \chi(s+1+n-1, s)=1
$$

Set

$$
G\left(m_{1} ; m, s\right)= \begin{cases}a\left(m_{1} ; s\right) v(m), & 0 \leq m \leq s \leq b-n \\ a\left(m_{1} ; s\right) v(m)+\chi(m, s), & 0 \leq s+1 \leq m \leq b\end{cases}
$$


Force $G$ to satisfy the nonlocal condition $P_{0}\left(m_{1}\right) u\left(m_{1}\right)=P_{0}(b) u(b)$; in particular, solve algebraically for $a\left(m_{1} ; s\right)$ to obtain

$$
\begin{aligned}
& a\left(m_{1} ; s\right)=\frac{-P_{0} \chi(b, s)}{P_{0} v(b)-P_{0} v\left(m_{1}\right)}, \quad m_{1} \leq s, \\
& a\left(m_{1} ; s\right)=\frac{P_{0} \chi\left(m_{1}, s\right)-P_{0} \chi(b, s)}{P_{0} v(b)-P_{0} v\left(m_{1}\right)}, \quad s+1 \leq m_{1} .
\end{aligned}
$$

Note that the right $(n-1 ; 1)$ disfocality implies that $P_{0} v(b)-P_{0} v\left(m_{1}\right)$ is nonzero; in particular, $a\left(m_{1} ; s\right)$ is well defined. Straightforward calculations show that

$$
\sum_{s=0}^{b-n} G\left(m_{1} ; m, s\right) f(s)
$$

is the unique solution of a nonhomogeneous BVP of the form $P u(m)=f(m), m \in$ $\{0, \ldots, b-n\}$, satisfying the boundary conditions (2.3).

Theorem 2.3. Assume that $P u=0$ is right $(n-1 ; 1)$ disfocal on $\{0, \ldots, b\}$. Then

$$
G\left(m_{1} ; m, s\right) \leq 0
$$

$\left(m_{1}, m, s\right) \in\{n-2, \ldots, b-1\} \times\{n-1, \ldots, b\} \times\{0, \ldots, b-n\}$. The inequality is strict, except in the conjugate case, $m_{1}=n-2$, at $m=b$.

Remark 2.4. We consider a specific set of nonlocal boundary conditions in this paper to illustrate that theory and methods from disconjugacy theory apply to families of nonlocal BVPs. Because of the specific nonlocal boundary conditions, it is the case that $P_{1} u$ has a generalized zero in $\left\{m_{1}, \ldots, b-1\right\}$. Hence, the argument we produce below is precisely the general argument for the conjugate boundary conditions given in [6, Section 8.8], after Rolle's theorem has been applied one time.

Proof. It is known that (2.9) is valid in the extreme cases, $m_{1}=n-2$ [15] and $m_{1}=b-1$ [10]. Let $m_{1} \in\{n-1, \ldots, b-2\}$ be fixed. We first show that $G$ is of fixed sign for

$$
(m, s) \in\{n-1, \ldots, b\} \times\{0, \ldots, b-n\} .
$$

Let $s \in\{0, \ldots, b-n\}$ be fixed. By construction, $G$, as a function of $m$, satisfies the boundary conditions (2.3).

Assume for the sake of contradiction that $G$ has an additional generalized zero at $m_{0}$ for some $m_{0} \in\{n-1, \ldots, b\}$. Then $P_{0} G$ takes on an additional generalized zero at $m_{0}$ since $v_{1}$ is of strict sign. Perform a count on the number of generalized zeros of each $P_{j} G$. (Since $m_{1}$ and $s$ are fixed, $P_{j} G$ is a function of $m$. We suppress the argument for simplicity of notation.) 
Note, from the boundary conditions, that $P_{1} G$ has a generalized zero at $n-3$. The first point of the argument is to argue that $P_{1} G$ has two more generalized zeros in $\{n-$ $2, \ldots, b-1\}$. First assume that $m_{0} \leq m_{1}$. Apply Rolle's theorem, Lemma 2.1. Then $P_{1} G$ has two more generalized zeros, $m_{11}<m_{12}$, where $m_{11} \in\left\{n-2, \ldots, m_{0}-1\right\}$ and $m_{12} \in$ $\left\{m_{1}, \ldots, b-1\right\}$. Second, assume that $m_{1}<m_{0}$. With this assumption, $P_{0} G\left(m_{1}\right) \neq 0$. Apply Rolle's theorem to see that $P_{1} G$ has a generalized zero at $m_{11} \in\left\{n-2, \ldots, m_{0}-1\right\}$. If $m_{11}<$ $m_{1}$, then Rolle's theorem can be applied to the nonlocal conditions to obtain a second generalized zero $m_{12} \in\left\{m_{1}, \ldots, b-1\right\}$. So, we come to the last subcase, $m_{1} \leq m_{11}$. Assume without loss of generality that $m_{0}$ is the smallest generalized zero of $P_{0} G$ to the right of $m_{1}$. Then $P_{0} G\left(m_{1}\right) P_{0} G\left(m_{0}\right) \leq 0$. This implies that $P_{0} G\left(m_{0}\right) P_{1} G\left(m_{0}-1\right) \geq 0$. These two inequalities imply that $P_{1} G$ has a generalized zero in $\left\{m_{0}, \ldots, b-1\right\}$. If not, then $\Delta P_{0} G$ has a fixed sign on $\left\{m_{0}, \ldots, b-1\right\}$, which agrees with the sign of $P_{1} G$ at $m_{0}-1$. Recall the identity

$$
P_{0} G(b)=P_{0} G\left(m_{0}\right)+\sum_{\mu=m_{0}}^{b-1} \Delta P_{0} G(\mu) .
$$

In particular, $P_{0} G\left(m_{1}\right)$ and $P_{0} G(b)$ have opposite signs which contradicts the nonlocal boundary conditions. Thus, there exists $m \in\left\{m_{0}, \ldots, b-1\right\}$ such that $P_{1} G\left(m_{0}-\right.$ 1) $P_{1} G(m) \leq 0$. In particular, there exists $m_{12} \in\left\{m_{0}, \ldots, b-1\right\}$ such that $P_{1} G$ has a generalized zero at $m_{12}$.

To summarize the purpose of the preceding paragraphs, we have shown that $P_{1} G$ has at least three generalized zeros on $\{n-3, \ldots, b-1\}$. It now easily follows by induction and repeated applications of Lemma 2.1 and the boundary conditions that for each $j=$ $0, \ldots, n-2 P_{j} G$ has at least 3 generalized zeros, one at $n-(j+2)$ and other two satisfying $n-(j+1) \leq m_{j 1}<m_{j 2} \leq b-j$.

Since $P_{n-2} G$ has at least three generalized zeros, $P_{n-2} G$ has at least two generalized zeros counting multiplicities for $m \leq s$ or for $s+1 \leq m$. Either case will provide a contradiction.

Assume that $P_{n-2} G$ has at least two generalized zeros counting multiplicities for $m \leq s$. Then $P_{n-1} G$ has at least one generalized zero for $m \leq s$. By construction, $P_{n} G \equiv 0$ for $t \leq s$; thus, $v_{n} P_{n-1} G$ is of constant sign and has a generalized zero; in particular, $P_{n-1} G \equiv 0$ for $m \leq s$. Continue inductively and argue that $P_{j} G \equiv 0$ for $m \leq s$. In particular, $G=v \equiv 0$ for $m \leq s$. This clearly contradicts the construction of $v$.

Assume that $P_{n-2} G$ has at least two generalized zeros counting multiplicities for $s+1 \leq$ $m$. Then a similar argument gives that $G=v+\chi \equiv 0$ for $s+1 \leq m$. If $v=-\chi$, then the disconjugacy is violated.

Thus, $G$ is of strict sign on $\{n-2, \ldots, b-1\} \times\{n-1, \ldots, b\} \times\{0, \ldots, b-n\}$.

To determine the sign of $G$, evaluate the sign of

$$
h(m)=\sum_{s=0}^{b-n} G(m, s),
$$


which is the unique solution of the BVP

$$
P u=1, \quad m \in\{0, \ldots, b-n\},
$$

with boundary conditions (2.3). $P_{j} h$ has a generalized zero at $n-(j+2)$ because of the boundary conditions. In addition, because of the nonlocal boundary conditions and repeated applications of Rolle's theorem, $P_{j} h$ has a generalized zero at $m_{j, 1}$, where

$$
n-(j+2)<m_{j, 1}<m_{j-1,1}<b
$$

for $j=1, \ldots, n-2$. Moreover, due to Rolle's theorem, $P_{n-1} h$ has precisely one generalized zero since $P_{n} u \equiv 1$.

$P_{n} u \equiv 1$ implies that $v_{n} P_{n-1} u$ is increasing. From the above construction, $v_{n} P_{n-1} u$ has precisely one generalized zero at $0<m_{n-1,1}$. Hence, $v_{n} P_{n-1} u<0$ on $\left\{0, \ldots, m_{n-1,1}-1\right\}$. Continue inductively. Initially, $v_{n-1} P_{n-2} u$ is decreasing and $P_{n-2} u(0)=0$; so $P_{n-2} u(1)<0$. Inductively, it follows that $P_{j} u(n-1-j)<0, j=0, \ldots, n-2$. In particular, $u(n-1)<0$; since $G$ does not change sign, $u$ does not change sign. Thus, $u$ negative implies that (2.9) is valid.

Theorem 2.5. Assume that $P u=0$ is right $(n-1 ; 1)$ disfocal on $\{0, \ldots, b\}$. Then $G$, as a function of $m_{1}$, is decreasing; that is, if $n-2 \leq m_{1}<m_{2} \leq b-1$, then

$$
G\left(m_{2} ; m, s\right)<G\left(m_{1} ; m, s\right) \leq 0,
$$

$(m, s) \in\{n-1, \ldots, b\} \times\{0, \ldots, b-n\}$. The second inequality is strict, except in the conjugate case, $m_{1}=n-2$, at $m=b$.

To prove the above comparison theorem, we first obtain a useful lemma. Let $G_{2}$ denote the quasidifference of $G$ with respect to $m$; that is, let

$$
G_{2}\left(m_{1} ; m, s\right)=P_{0} G\left(m_{1} ; m+1, s\right)-P_{0} G\left(m_{1} ; m, s\right)=v_{2} P_{1} G
$$

Lemma 2.6. Let $m_{1} \in\{n-2, \ldots, b-2\}$. Then

$$
G_{2}\left(m_{1}+1 ; m_{1}, s\right)<0, \quad s \in\{0, \ldots, b-n\}
$$

Proof. The proof requires only a simple extension from the proof of Theorem 2.3. As summarized in the fourth paragraph of the proof of Theorem 2.3, we know that $P_{1} G$ has precisely one generalized zero $m_{11}$ to the right of $n-3$. We also know by Rolle's theorem that $m_{1} \leq m_{11}$.

$G(n-2)=0, G(n-1)<0$ imply that $P_{1} G(n-2)<0$ which in turn implies that $P_{1} G\left(m_{1}-1\right)<0$.

Proof of Theorem 2.5. Let $G_{1}$ denote the difference of $G$ with respect to $m_{1}$; that is, let

$$
G_{1}\left(m_{1} ; m, s\right)=G\left(m_{1}+1 ; m, s\right)-G\left(m_{1} ; m, s\right) \text {. }
$$

In particular, we assume that $m_{1}<b-1$. 
Note that $G_{1}$ is the unique solution of the BVP

$$
\begin{gathered}
P u=0, \quad m \in\{0, \ldots, b-n\}, \\
P_{j} u(0)=0, \quad j=0, \ldots, n-2, \\
P_{0} u(b)-P_{0} u\left(m_{1}\right)=G_{2}\left(m_{1}+1 ; m_{1}, s\right)<0 .
\end{gathered}
$$

$G_{1}$ satisfies the difference equation and each of the initial conditions at 0 ; this is clear since each term of $G\left(m_{1}+1 ; m, s\right), G\left(m_{1} ; m, s\right)$ satisfies the difference equation and the initial conditions. Simply calculate the nonlocal boundary condition

$$
\begin{aligned}
P_{0}(G( & \left.\left.m_{1}+1 ; b, s\right)-G\left(m_{1} ; b, s\right)\right)-P_{0}\left(G\left(m_{1}+1 ; m_{1}, s\right)-G\left(m_{1} ; m_{1}, s\right)\right) \\
= & \left(P_{0} G\left(m_{1}+1 ; b, s\right)-P_{0} G\left(m_{1}+1 ; m_{1}+1, s\right)\right) \\
& +\left(P_{0} G\left(m_{1}+1 ; m_{1}+1, s\right)-P_{0} G\left(m_{1}+1 ; m_{1}, s\right)\right) \\
= & G_{2}\left(m_{1}+1 ; m_{1}, s\right) .
\end{aligned}
$$

In particular,

$$
P_{0} u(b)<P_{0} u\left(m_{1}\right) \text {. }
$$

The boundary conditions at 0 and the right $(n-1 ; 1)$ disfocality imply that $P_{0} u$ is monotone for $m>n-2 . P_{0} u(b)<P_{0} u\left(m_{1}\right)$ implies that $P_{0} u$ is monotone-decreasing and (2.15) is proved.

We end the paper with a brief general observation. Let $l \in\{0, \ldots, n-2\}$. Let $m_{1} \in$ $\{n-2, \ldots, b-l-1\}$. Consider the BVP

$$
P u(m)=0, \quad m \in\{0, \ldots, b-n\},
$$

with boundary conditions

$$
P_{j} u(0)=0, \quad j=0, \ldots, n-2, \quad P_{l} u\left(m_{1}\right)=P_{l} u(b-l) .
$$

We state without proof theorems analogous to Theorems 2.3 and 2.5. The observation to make now is that the BVP at $l=0, m_{1}=b-1$ is equivalent to the BVP with $l=1$, $m_{1}=n-3$. One can now begin an inductive argument on $l$ and repeat the arguments in the paper.

A Green function $G\left(l, m_{1} ; m, s\right)$ for the BVP $(1.1),(2.23)$ is readily constructed as in the proof of Theorem 2.2. So, from the above observation, we claim that

$$
G(0, b-1 ; m, s)=G(1, n-3 ; m, s)
$$

Define the $j$ th difference of $G$ with respect to $m$ by $\Delta_{j} G$. The proof of Theorem 2.3 generalizes readily to show that

$$
\Delta_{l} G\left(l, m_{1} ; m, s\right) \leq 0,
$$


$\left(m_{1}, m, s\right) \in\{n-l-2, \ldots, b-l-1\} \times\{n-l-1, \ldots, b-l\} \times\{0, \ldots, b-n\}$. We do not present the proofs because the arguments, applied to $\Delta_{l} G\left(l, m_{1} ; m, s\right)$, go through in complete analogy; the inequalities for the lower-order differences are then obtained through repeated definite summations from $m=0$, which are valid because of the boundary conditions.

Theorem 2.7. Assume that $P u=0$ is right $(n-1 ; l)$ disfocal on $\{0, \ldots, b\}$. Then

$$
\Delta_{j} G\left(l, m_{1} ; m, s\right) \leq 0
$$

$\left(m_{1}, m, s\right) \in\{n-2, \ldots, b-l-1\} \times\{n-j-1, \ldots, b-j\} \times\{0, \ldots, b-n\}, j=0, \ldots, l$. The inequality is strict, except in the case $j=l, m_{1}=n-l-2$, at $m=b-l$.

Theorem 2.8. Assume that $P u=0$ is right $(n-1 ; l)$ disfocal on $\{0, \ldots, b\}$. Then $\Delta_{j} G$, as a function of $m_{1}$, is decreasing; that is, if $n-2 \leq m_{1}<m_{2} \leq b-l-1$, then

$$
\Delta_{j} G\left(l, m_{2} ; m, s\right)<\Delta_{j} G\left(l, m_{1} ; m, s\right) \leq 0,
$$

$(m, s) \in\{n-j-1, \ldots, b-j\} \times\{0, \ldots, b-n\}, j=0, \ldots, l$. The second inequality is strict, except in the case $j=l, m_{1}=n-l-2$, at $m=b-l$.

Finally, in the spirit of the interesting comparison theorems first introduced by Elias [7] (see also [19] or [11]) and later discretized [10], we close with the following comparison theorem.

Theorem 2.9. Assume that $P u=0$ is right-disfocal on $\{0, \ldots, b\}$. Let $l_{1}<l_{2}$. Then

$$
\Delta_{j} G\left(l_{2}, m_{l_{2}} ; m, s\right)<\Delta_{j} G\left(l_{1}, m_{l_{1}} ; m, s\right) \leq 0
$$

$(m, s) \in\{n-j-1, \ldots, b-j\} \times\{0, \ldots, b-n\}, j=0, \ldots, l_{1}$. The inequality is strict, except in the case $j=l_{1}, m_{1}=n-l_{1}-2$, at $m=b-l_{1}$. If $l_{1}=l_{2}$ and $m_{1}<m_{2}$, then $\Delta_{j} G\left(l, m_{2} ; m, s\right)<$ $\Delta_{j} G\left(l, m_{1} ; m, s\right) \leq 0,(m, s) \in\{n-j-1, \ldots, b-j\} \times\{0, \ldots, b-n\}, j=0, \ldots, l_{1}=l_{2}$. The inequality is strict, except in the case, $j=l_{1}, m_{1}=n-l_{1}-2$, at $m=b-l_{1}$.

\section{References}

[1] R. P. Agarwal, Difference Equations and Inequalities, Monographs and Textbooks in Pure and Applied Mathematics, vol. 155, Marcel Dekker, New York, 1992.

[2] __ Focal Boundary Value Problems for Differential and Difference Equations, Mathematics and Its Applications, vol. 436, Kluwer Academic Publishers, Dordrecht, 1998.

[3] A. V. Bitsadze, On the theory of nonlocal boundary value problems, Soviet Math. Dokl. 30 (1984), $8-10$.

[4] A. V. Bitsadze and A. A. Samarskii, On some simple generalizations of linear elliptic boundary problems, Soviet Math. Dokl. 10 (1969), 398-400.

[5] L. E. Bobisud, Three-point boundary value problems for some nonlinear second-order differential equations, Dynam. Systems Appl. 10 (2001), no. 4, 489-516.

[6] M. Bohner and A. Peterson, Advances in Dynamic Equations on Time Scales, Birkhäuser Boston, Massachusetts, 2003.

[7] U. Elias, Green's functions for a nondisconjugate differential operator, J. Differential Equations 37 (1980), no. 3, 318-350. 
[8] P. W. Eloe, Criteria for right disfocality of linear difference equations, J. Math. Anal. Appl. 120 (1986), no. 2, 610-621.

[9] P. W. Eloe and J. Henderson, Focal points and comparison theorems for a class of two-point boundary value problems, J. Differential Equations 103 (1993), no. 2, 375-386.

[10] P. W. Eloe and E. R. Kaufmann, A singular boundary value problem for a right disfocal linear differential operator, Dynam. Systems Appl. 5 (1996), no. 2, 175-182.

[11] P. W. Eloe and J. Ridenhour, Sign properties of Green's functions for a family of two-point boundary value problems, Proc. Amer. Math. Soc. 120 (1994), no. 2, 443-452.

[12] T. Fort, Finite Differences and Difference Equations in the Real Domain, Clarendon Press, Oxford, 1948.

[13] C. P. Gupta, Solvability of a three-point nonlinear boundary value problem for a second order ordinary differential equation, J. Math. Anal. Appl. 168 (1992), no. 2, 540-551.

[14] C. P. Gupta and S. I. Trofimchuk, A sharper condition for the solvability of a three-point second order boundary value problem, J. Math. Anal. Appl. 205 (1997), no. 2, 586-597.

[15] P. Hartman, Difference equations: disconjugacy, principal solutions, Green's functions, complete monotonicity, Trans. Amer. Math. Soc. 246 (1978), 1-30.

[16] V. A. Il'in and E. I. Moiseev, Nonlocal boundary value problems of the first kind for a SturmLiouville operator in its differential and finite difference aspects, Differ. Equ. 23 (1987), no. 7, 803-810.

[17] Nonlocal boundary value problems of the second kind for a Sturm-Liouville operator, Differ. Equ. 23 (1987), no. 8, 979-987.

[18] A. Lomtatidze, On a nonlocal boundary value problem for second order linear ordinary differential equations, J. Math. Anal. Appl. 193 (1995), no. 3, 889-908.

[19] A. Peterson and J. Ridenhour, Comparison theorems for Green's functions for focal boundary value problems, Recent Trends in Differential Equations, World Sci. Ser. Appl. Anal., vol. 1, World Scientific Publishing, New Jersey, 1992, pp. 493-506.

Paul W. Eloe: Department of Mathematics, University of Dayton, Dayton, OH 45469-2316, USA E-mail address: paul.eloe@notes.udayton.edu 